\title{
Revealing and quantifying the impaired phonological analysis underpinning impaired comprehension in Wernicke's aphasia
}

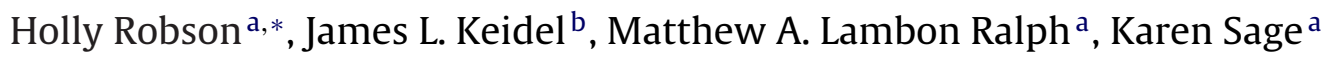 \\ a Neuroscience and Aphasia Research Unit, University of Manchester, United Kingdom \\ ${ }^{\mathrm{b}}$ School of Psychology, Bangor University, United Kingdom
}

\section{A R T I C L E I N F O}

\section{Article history:}

Received 2 March 2011

Received in revised form 7 November 2011

Accepted 29 November 2011

Available online 8 December 2011

\section{Keywords:}

Wernicke's Aphasia

Comprehension

Phonology

Semantics

Auditory discrimination

\begin{abstract}
A B S T R A C T
Wernicke's aphasia is a condition which results in severely disrupted language comprehension following a lesion to the left temporo-parietal region. A phonological analysis deficit has traditionally been held to be at the root of the comprehension impairment in Wernicke's aphasia, a view consistent with current functional neuroimaging which finds areas in the superior temporal cortex responsive to phonological stimuli. However behavioural evidence to support the link between a phonological analysis deficit and auditory comprehension has not been yet shown. This study extends seminal work by Blumstein, Baker, and Goodglass (1977) to investigate the relationship between acoustic-phonological perception, measured through phonological discrimination, and auditory comprehension in a case series of Wernicke's aphasia participants. A novel adaptive phonological discrimination task was used to obtain reliable thresholds of the phonological perceptual distance required between nonwords before they could be discriminated. Wernicke's aphasia participants showed significantly elevated thresholds compared to age and hearing matched control participants. Acoustic-phonological thresholds correlated strongly with auditory comprehension abilities in Wernicke's aphasia. In contrast, nonverbal semantic skills showed no relationship with auditory comprehension. The results are evaluated in the context of recent neurobiological models of language and suggest that impaired acoustic-phonological perception underlies the comprehension impairment in Wernicke's aphasia and favour models of language which propose a leftward asymmetry in phonological analysis.
\end{abstract}

(c) 2011 Elsevier Ltd. All rights reserved.

\section{Introduction}

\subsection{Wernicke's aphasia: the neuropsychological profile}

Wernicke's aphasia (WA) is a relatively rare condition principally resulting from a cerebral vascular accident (CVA) to the posterior temporoparietal cortex. WA is characterised by severely impaired auditory comprehension and repetition, with fluent speech punctuated by phonological paraphasias and neologisms (Goodglass, Kaplan, \& Barresi, 2001a). Other linguistic skills, such as reading and writing, are variable but rarely spared (e.g. Ellis, Miller, $\& \operatorname{Sin}, 1983)$. A comprehension impairment post stroke is not, however, synonymous with WA. Up to $70 \%$ of individuals with aphasia display some degree of comprehension impairment at a sentence level (Boller, Kim, \& Mack, 1977) but only a small proportion of these fulfil the diagnosis for WA. Crucially, the comprehension

\footnotetext{
* Corresponding author at: Neuroscience and Aphasia Research Unit (NARU), Zochonis Building, School of Psychological Sciences, University of Manchester, Oxford Road, Manchester, M13 9PL, UK. Tel.: +44 1612752683.

E-mail addresses: holly.robson@manchester.ac.uk (H. Robson), karen.sage@manchester.ac.uk (K. Sage).
}

impairment in WA is found at a single word level and co-occurs with both fluent speech and impaired repetition. The exclusion of any of the three diagnostic criteria will result in a different aphasia subtype (e.g. global aphasia, conduction aphasia or transcortical sensory aphasia: Goodglass et al., 2001a,b).

The traditional account of the WA auditory comprehension impairment proposes disruption to acoustic-phonological decoding as the underlying deficit (Eggert, 1977; Luria, 1976). This has been termed the "decoding deficit" (Luria, 1976) as it proposes an impairment of auditory analysis of phonemes but a sparing of auditory analysis of prosody, leading to an apparent dissociation between comprehension in formal testing conditions and contextual comprehension. In everyday conversation this dissociation manifests as appropriate social responses to intonation, however, the capacity to respond to specific linguistic information in context remains impaired. The decoding deficit is consistent with reports that lesions in WA consistently affect the posterior part of the left superior temporal plane (Bogen \& Bogen, 1976), a region associated with phonological processing (Vigneau et al., 2006). A discrepancy between formal and contextual comprehension is not unique to WA however; such a pattern may also be explained by the use of contextual information to support comprehension. A second account proposes that impaired semantic processing underlies impaired 
comprehension behaviours in WA (Gainotti, Miceli, Silveri, \& Villa, 1982; Hickok, 2000). This view, in turn, is supported by recent reports which indicate posterior middle temporal areas, related to semantic processing, as the site of highest lesion overlap in WA (Dronkers, Wilkins, Van Valin, Redfern, \& Jaeger, 2004; Vigneau et al., 2006).

\subsection{Investigations into the comprehension impairment in WA}

A recent case series study of nine participants with a clinical diagnosis of WA (using the diagnostic criteria outlined above) found that all participants had an acoustic-phonological impairment and that, in the majority of participants, this was combined with a non-verbal semantic impairment (Robson et al., in press). Six out of nine participants were impaired on a non-verbal pictorial association task (The Pyramids and Palm Trees test: Howard \& Patterson, 1992), where three pictures are displayed and participants must identify an association between the probe picture (pyramid) and the target picture (palm tree), ignoring the distractor picture (pine tree). When the same assessment was carried out using written words instead of pictures the participants' accuracy significantly decreased. A similar pattern was noted in word to picture matching tests; spoken word-picture matching led to a significant decline in accuracy compared to written word-picture matching of the same items. Overall, these results suggested that access to semantic knowledge in WA was modulated by input modality of the testing materials; specifically that, as the need for acoustic-phonological analysis increased, accuracy decreased. It is, however, important to establish whether an impairment in acoustic-phonological analysis, measured in isolation from other linguistic and semantic knowledge, shows a direct relationship with auditory comprehension in WA.

\subsection{Acoustic-phonological processing in aphasia}

Direct investigations into acoustic-phonological processing have a substantial history in aphasiology. Such research primarily involves testing participants with phonological discrimination (judging whether two phoneme strings are the same or different) and phonological identification (matching auditory presented phonemes to a set of options) (Gow \& Caplan, 1996). Consistently, individuals with aphasia have been shown to be impaired at both phonological discrimination and identification tasks (e.g. Basso, Casati, \& Vignolo, 1977; Baum, 2002; Blumstein et al., 1977; Gainotti et al., 1982; Leeper, Shewan, \& Booth, 1986; Miceli, Arena, Caltagirone, Gianotti, \& Payer-Rigo, 1977; Miceli, Gainotti, Caltagirone, \& Masullo, 1980; Tallal \& Newcombe, 1978). Although such acoustic-perceptual impairments are not a universal feature of aphasia (Varney, 1984) they appear to occur commonly among all subtypes including WA (Blumstein et al., 1977; Csepe, OsmanSagi, Molnar, \& Gosy, 2001), Broca's aphasia (Csepe et al., 2001; Leeper et al., 1986), conduction aphasia (Leeper et al., 1986) and global aphasia (Varney, 1984). One particularly interesting finding is that acoustic-phonological processing impairments are not simply a consequence of a broader brain damage effect. Individuals with non-aphasic left hemisphere and right hemisphere neural injury do not show the same degree of impairment as aphasic left hemisphere neural injury and, in many cases, these individuals are not found to be significantly different to control participants (Basso et al., 1977; Miceli et al., 1977). This indicates that areas involved in acoustic-phonological processing tasks also subserve the wider language network.

\subsection{Acoustic-phonological disorders and language comprehension}

Despite the intuitive link between acoustic-phonological processing abilities and language comprehension, behavioural evidence supporting this link has been inconsistent. Some studies of mixed groups of aphasia participants, some with large cohorts, have failed to find a link between acousitic-phonological processing and comprehension (Basso et al., 1977; Jauhiainen \& Nuutila, 1977). Others have had more success; Tallal and Newcombe (1978) found a relationship between CV sequence identification and sentence comprehension and Miceli et al. (1980) found a relationship between phonological discrimination and sentence comprehension in a large group of aphasic participants. Additionally it has been claimed that improvement in auditory comprehension and phonological discrimination after stroke go hand-in-hand (Varney, 1984).

An attempt to establish a direct relationship between impaired acoustic-phonological analysis and auditory comprehension in WA was undertaken by Blumstein et al. (1977). This study used a case series comparison to test the relationship between the ability to discriminate whether pairs of words or nonwords were the same or different and auditory comprehension scores measured via the Boston Diagnostic Aphasia Examination (Goodglass \& Kaplan, 1972). Pairs of one and two syllables were used; each of the different pairs differed by one English stop phoneme ( $\mathrm{p} \mathrm{t} \mathrm{b} \mathrm{d} \mathrm{k} \mathrm{g).} \mathrm{The}$ study investigated 25 participants with aphasia who were subdivided into four clinical groups: Wernicke's aphasia $(n=6)$, mixed posterior lesions $(n=7)$, Broca's aphasia $(n=6)$ and mixed anterior lesions $(n=6)$. Including all participants in the analysis, a significant correlation between auditory comprehension and phonological discrimination was established. The authors indicated however that this correlation was driven by the superior performance of the individuals with Broca's aphasia in both tasks. The WA were the only group where discrimination accuracy appeared to change systematically with comprehension scores. However this finding did not reach significance and they were unable to conclude from these data whether the impairment in phonemic hearing was sufficient to account for the comprehension impairment in WA. Instead, Blumstein et al. (1977) suggested that it plays a contributory (rather than a primary) role in the participants' comprehension impairment. It is possible, however, that Blumstein et al.'s inability to demonstrate a strong link between acoustic-phonological analysis and comprehension was due to the small numbers of WA participants $(n=6)$ included in the analysis, as suggested by the authors.

Gainotti et al. (1982), in a study similar to Blumstein et al. (1977), investigated a larger group of 22 WA patients and found a similarly weak link between acoustic-phonological analysis and comprehension. However, in this study, inclusion criteria for diagnosis of WA were presence of fluent speech and moderate to severe disorders of phrase comprehension. Crucially, they did not require participants to have impaired repetition, which is integral to the diagnosis of classical WA (Goodglass et al., 2001a). In addition, the comprehension impairment was only required at a phrase rather than a single word level. Lesion data from CT scans indicated that just $12 / 22$ of the participants displayed involvement of the posterior temporal lobe. It is possible, therefore, that the advantage of increased participant numbers was negated by an over-inclusive diagnostic criteria; Gainotti et al.'s (1982) study may have included a mix of other aphasia subtypes (e.g. transcortical sensory aphasia) within its umbrella of WA group. If, for example, a proportion of the patients had transcortical sensory aphasia (comprehension impairment without phonological and repetition deficits) then, by definition, any relationship between phonological impairment and comprehension disorder in the WA subset could be washed out.

The null results obtained in these two previous studies have been highly influential in the aphasiological and neuroscience fields. The lack of significant results linking phonological analysis to comprehension in combination with the failure of individuals with WA on semantic tasks (e.g. Cohen, Kelter, \& Woll, 1980; De Renzi, Faglioni, Scotti, \& Spinnler, 1972; Gainotti, Silveri, Villa, \& Caltagirone, 1983) has led to the interpretation that the primary 
locus of the impairment in WA is at a post-phonological level either in the mapping between sound and meaning (Hickok, 2000, Baker, Blumstein, \& Goodglass, 1981) or to semantic representations themselves (Binder et al., 2000; Gainotti et al., 1982). This, in turn, appears to have influenced the development of neurobiological models of language and interpretation of the function of areas damaged in WA (Hickok, 2000; Hickok \& Poeppel, 2004, 2007) and potentially could influence decisions made in evidence based clinical remediation. Given the importance that has been placed on these null results, it is important to revisit the question of the underlying deficit in WA and to establish strong evidence upon which further research can be based.

The current study aimed to revisit the seminal works on acoustic-phonological discrimination in WA conducted by Blumstein et al. (1977), Gainotti et al. (1982) and others with larger number of participants $(n=11)$, each with carefully defined WA in order to examine whether the observed deficit in acoustic-phonological processing varied systematically with their comprehension impairment. The study did not therefore include everyone who showed impaired comprehension; rather it recruited only those who met the following three key diagnostic features of classical WA: impaired comprehension at a single word level; impaired single word repetition and fluent but semantically empty speech. To further improve statistical power, a sensitive probe of phonological discrimination was used which allowed the variation in the degree of phonological discrimination impairment to be observed across the participants.

\section{Participants}

\subsection{Ethical approval}

Approval for this research was granted by the regional ethics committee. Eleven participants with a diagnosis of WA and eleven matched control participants were recruited.

\subsection{WA participants}

Participants with WA were recruited from speech and language therapy services in the North of England and all participants gave informed consent. Seven of the eleven participants had participated in a previous study (Robson, Sage, \& Lambon Ralph, in press). Biographical information and background assessments are displayed in Table 1 . The recruitment criteria given to speech and language therapists was the classical WA profile, i.e. fluent speech, impaired single word comprehension and errors on repetition or naming, the error type was not specified. All participants were screened to confirm diagnosis using the Boston Diagnostic Aphasia Examination - 3rd edition (BDAE: Goodglass, Kaplan, \& Barresi, 2001b). BDAE profiles are displayed in Fig. 1. All but two participants ( $\mathrm{CW}$ and $\mathrm{CH}$ ) were below the bottom 20th centile on the auditory comprehension subtests of the BDAE (Table 1). All participants had severely impaired repetition and fluent speech punctuated with multiple phonological paraphasias and neologistic errors. Semantic paraphasias were rare. Reading and writing were impaired for all participants. Fluency was defined according to the BDAE - 3rd edition (see Fig. 1) over the parameters of articulatory agility, grammatical form, phrase length and melodic line. Both the cookie theft picture description and conversational and expository speech were taken into account in this judgment. All but one participant (CB) were fully mobile; hemiplegia is relatively uncommon in WA because their posterior lesions do not often extend into motor areas. Patient $C B$ was an exception as his lesion did extend into motor regions. Eight of the participants displayed sensori-neural hearing loss commensurate with their age (Gates, Cooper, Kannel, \& Miller, 1990).

\subsection{WA participant neuroimaging}

Chronic MRI T1w scans (high resolution, 3D structural brain images) were available for eight of the eleven participants (DL, LB, CB, RD, DM, EL, CH, CW) from which a lesion overlap map could be created; for the remaining three participants only acute CT scans could be acquired. T1w images were acquired on a 3 T Philips Achieva scanner with an eight element SENSE head coil with a sense factor of 2.5. An inversion recovery sequence produced a $256 \times 256$ matrix of 128 transverse slices with $1 \mathrm{~mm}^{3}$ voxels. Automated lesion identification was carried out on the eight MR images using the procedure described in Seghier, Ramlackhansingh, Crinion, Leff, and Price (2008). This algorithm enhances lesion identification by adding an extra tissue class to the unified segmentation algorithm (Ashburner \& Friston, 2005) representing the lesion. Following segmentation, grey matter (GM) and white matter (WM) images were then smoothed using a $8 \mathrm{~mm}$ full-width half maximum Gaussian kernel. After smoothing, GM and WM lesions for each participant were identified using the lesion detection algorithm. Outlier images were produced which identified the degree of abnormality on a voxel-by-voxel basis in GM and WM images in comparison to 13 elderly healthy control participants; voxels with a degree of abnormality greater than 0.5 were identified as lesion. These lesion images were combined to create a lesion overlap map and displayed on a standard MNI brain template using the MRIcro software (Rorden, 2005), see Fig. 2. Maximal lesion overlap was observed at the left temporoparietal junction which is consistent with traditional accounts of the lesion in Wernicke's aphasia (Bogen \& Bogen, 1976). Analysis of CT scans for the remaining three participants

Table 1

Background information and assessment results for the WA participants.

\begin{tabular}{|c|c|c|c|c|c|c|c|c|c|c|c|}
\hline \multirow[t]{2}{*}{ Participant } & \multirow[t]{2}{*}{ Age } & \multirow[t]{2}{*}{ Sex } & \multirow{2}{*}{$\begin{array}{l}\text { Time post-onset } \\
\text { at testing }\end{array}$} & \multicolumn{4}{|c|}{ BDAE comprehension } & \multirow{2}{*}{$\begin{array}{l}\text { BDAE fluency } \\
\text { Percentile }\end{array}$} & \multicolumn{2}{|c|}{ BDAE repetition } & \multirow{2}{*}{$\begin{array}{l}\text { PPT } \\
\text { Raw: max } 52\end{array}$} \\
\hline & & & & $\begin{array}{l}\text { Total } \\
\text { Percentile }\end{array}$ & $\begin{array}{l}\text { Total } \\
\text { Raw: max } 32\end{array}$ & $\begin{array}{l}\text { sWPM } \\
\text { Raw: max } 16\end{array}$ & $\begin{array}{l}\text { Sentences } \\
\text { Raw: max } 16\end{array}$ & & $\begin{array}{l}\text { Sentence } \\
\text { Percentile }\end{array}$ & $\begin{array}{l}\text { Word } \\
\text { Percentile }\end{array}$ & \\
\hline DL & 73 & M & 6 months & 3 & 5 & 4 & 1 & 63 & $<1$ & $<1$ & 46 \\
\hline LB & 78 & $\mathrm{~F}$ & 7 years & 5 & 9 & 9 & 0 & 68 & 5 & 15 & 48 \\
\hline $\mathrm{CB}$ & 59 & M & 14 months & 10 & 8 & 5 & 3 & 38 & 15 & 10 & 42 \\
\hline MR & 64 & M & 20 months & 10 & 13.5 & 9.5 & 4 & 63 & 15 & 15 & 50 \\
\hline $\mathrm{RD}$ & 86 & M & 2 years & 10 & 14 & 12 & 2 & 80 & 5 & 10 & 50 \\
\hline $\mathrm{DH}$ & 74 & M & 3 years & 12 & 14 & 8 & 6 & 51 & 10 & 15 & 43 \\
\hline DM & 86 & $\mathrm{M}$ & 18 months & 13 & 15 & 8 & 7 & 57 & $<1$ & $<1$ & 39 \\
\hline EL & 61 & $\mathrm{M}$ & 19 months & 14 & 17 & 13 & 4 & 75 & 10 & 15 & 48 \\
\hline$A C$ & 53 & M & 3 years & 15 & 17.5 & 9.5 & 8 & 68 & $<1$ & $<1$ & 49 \\
\hline $\mathrm{CH}$ & 77 & $\mathrm{M}$ & 2 years & 40 & 26 & 14 & 12 & 90 & 45 & 5 & 51 \\
\hline $\mathrm{CW}$ & 70 & $\mathrm{M}$ & 3.5 years & 40 & 25 & 13 & 12 & 100 & 40 & 15 & 51 \\
\hline
\end{tabular}

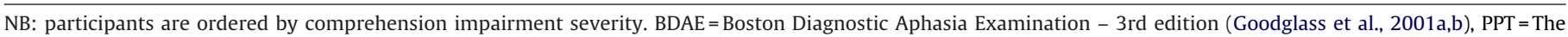
Pyramids and Palm Trees test (Howard \& Patterson, 1992). Normal performance for the PPT is between 49 and 52. 


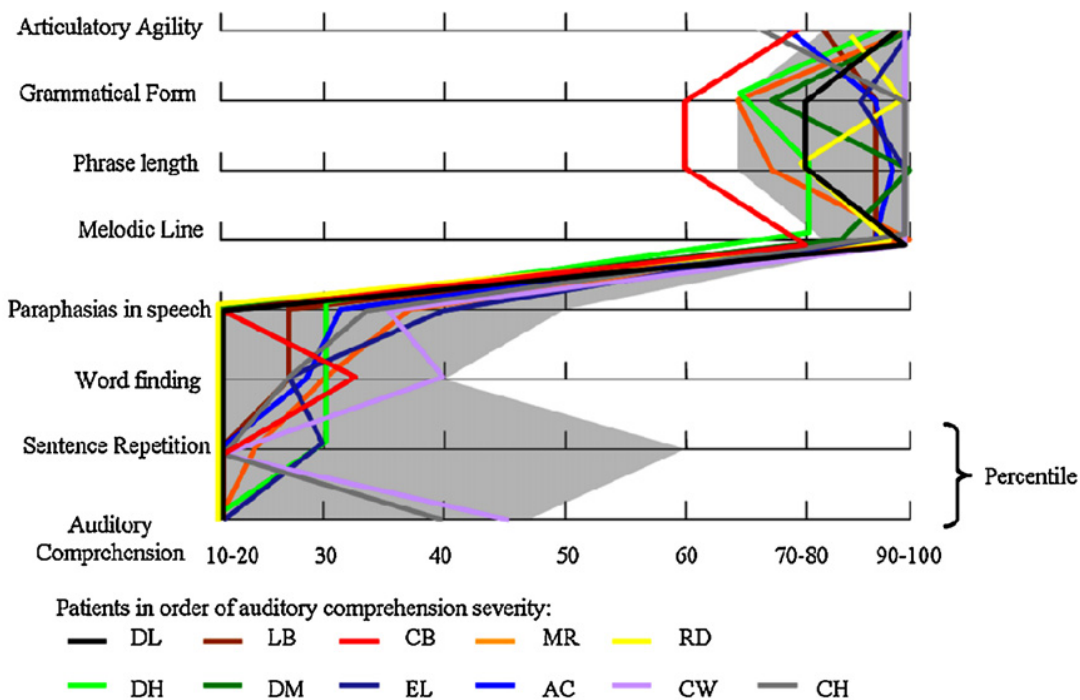

NB: Grey area represents area within which a participant should fall to be classified with Wernicke's aphasia

Fig. 1. Boston diagnostic aphasia examination profiles for Wernicke's aphasia participants.

were undertaken using templates for analysis of CT scans (Damasio \& Damasio, 1989). All three participants showed involvement of the superior temporal gyrus extending into the temporoparietal junction.

\subsection{Control participants}

Eleven age and hearing matched controls were recruited primarily from a database of elderly volunteers as well as relatives of the WA participants. All participants gave informed consent.
Control participants did not significantly differ from WA participants in age (Table 2 ).

\subsection{Pure tone hearing thresholds}

Pure tone hearing thresholds were measured for all WA and control participants. Thresholds were measured at $500 \mathrm{~Hz}, 1 \mathrm{kHz}$, $2 \mathrm{kHz}$ and $4 \mathrm{kHz}$ in the left and right ear in the environment in which the experiment was conducted, a quiet room in the participants' own homes. Participants did not wear hearing aids during

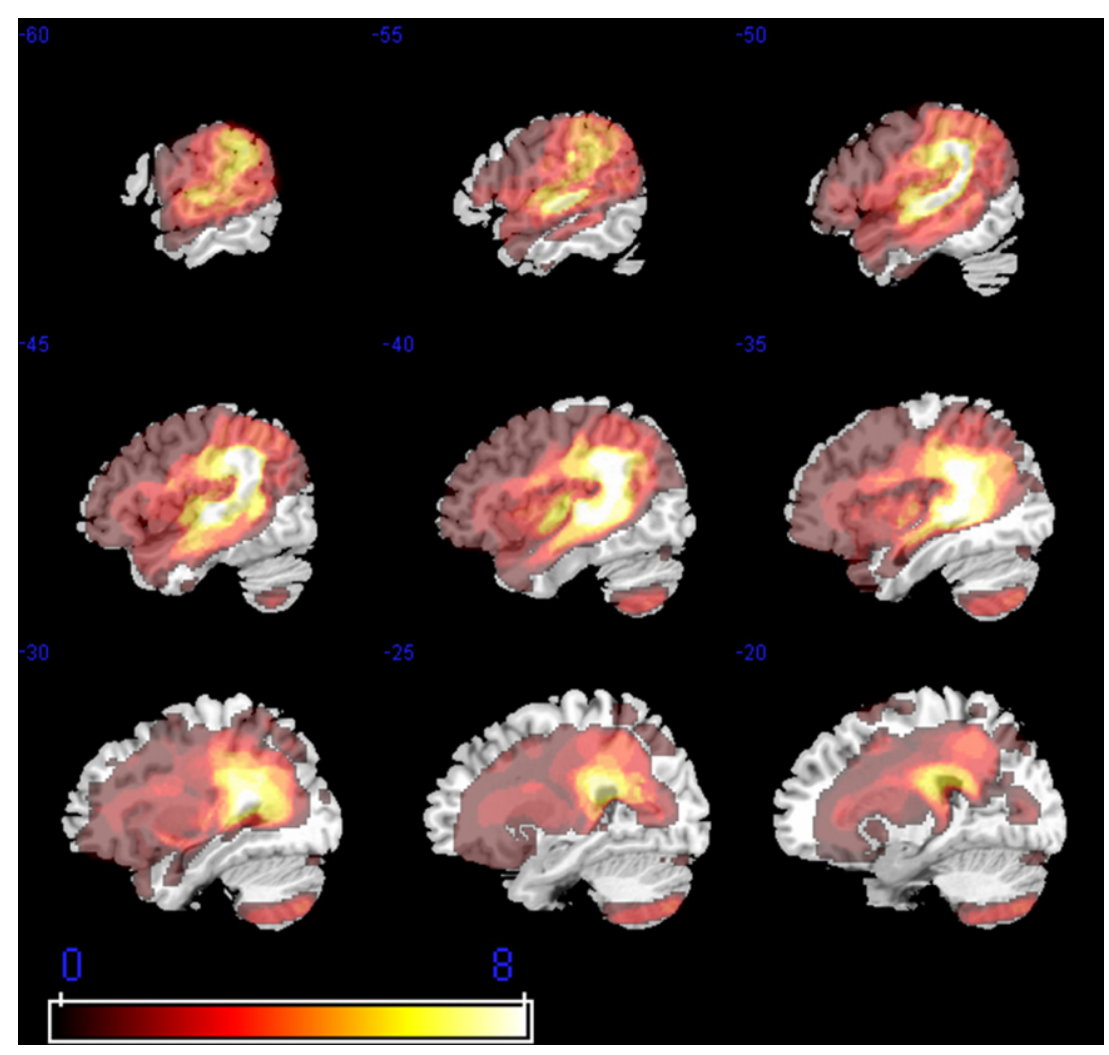

Fig. 2. Map of areas of abnormality in the Wernicke's aphasia participants. 
Table 2

Age and pure tone hearing thresholds for the WA and control participants.

\begin{tabular}{|c|c|c|c|c|c|c|c|c|c|c|c|}
\hline & \multirow[t]{2}{*}{ Participant } & \multirow[t]{2}{*}{ Age } & \multicolumn{4}{|c|}{ Left ear pure tone thresholds $\mathrm{dB}$} & \multicolumn{4}{|c|}{ Right ear pure tone thresholds $\mathrm{dB}$} & \multirow[t]{2}{*}{ Overall hearing factor } \\
\hline & & & $500 \mathrm{~Hz}$ & $1 \mathrm{kHz}$ & $2 \mathrm{kHz}$ & $4 \mathrm{kHz}$ & $500 \mathrm{~Hz}$ & $1 \mathrm{kHz}$ & $2 \mathrm{kHz}$ & $4 \mathrm{kHz}$ & \\
\hline \multirow[t]{12}{*}{ Wernicke's aphasia } & DL & 73 & 30 & 15 & 10 & 15 & 30 & 15 & 15 & 20 & -0.7 \\
\hline & LB & 80 & 10 & 10 & 30 & 40 & 5 & 20 & 15 & 10 & -0.83 \\
\hline & $\mathrm{CB}$ & 59 & 15 & 10 & 5 & 30 & 15 & 15 & 5 & 20 & -1.14 \\
\hline & MR & 66 & 35 & 50 & 45 & 60 & 25 & 10 & 15 & 25 & 0.51 \\
\hline & $\mathrm{RD}$ & 87 & 25 & 20 & 65 & 60 & 30 & 20 & 50 & 65 & 1.06 \\
\hline & $\mathrm{DH}$ & 74 & 5 & 5 & 30 & 45 & 15 & 10 & 30 & 50 & -0.57 \\
\hline & $\mathrm{DM}$ & 70 & 15 & 10 & 15 & 40 & 10 & 15 & 25 & 60 & -0.49 \\
\hline & EL & 61 & 20 & 25 & 50 & 65 & 15 & 30 & 45 & 65 & 0.94 \\
\hline & $A C$ & 54 & 10 & 15 & 5 & 20 & 10 & 20 & 20 & 50 & -0.82 \\
\hline & $\mathrm{CH}$ & 77 & 10 & 10 & 15 & 40 & 10 & 20 & 20 & 50 & -0.60 \\
\hline & $\mathrm{CW}$ & 70 & 45 & 50 & 60 & 70 & 25 & 35 & 55 & 75 & 2.15 \\
\hline & Mean & 70.1 & 20.0 & 20.0 & 30.0 & 44.1 & 17.3 & 19.1 & 26.8 & 44.5 & -0.04 \\
\hline \multirow[t]{11}{*}{ Controls } & DW & 73 & 25 & 15 & 25 & 45 & 25 & 15 & 20 & 40 & -0.19 \\
\hline & $\mathrm{PB}$ & 80 & 15 & 20 & 55 & 70 & 15 & 20 & 55 & 65 & 0.83 \\
\hline & PD & 60 & 15 & 15 & 15 & 35 & 50 & 15 & 15 & 55 & -0.25 \\
\hline & HW & 67 & 25 & 10 & 10 & 15 & 15 & 20 & 5 & 30 & -0.93 \\
\hline & $\mathrm{TA}$ & 84 & 20 & 10 & 20 & 40 & 25 & 10 & 20 & 70 & -0.31 \\
\hline & $\mathrm{HE}$ & 76 & 25 & 25 & 50 & 70 & 20 & 15 & 40 & 60 & 0.74 \\
\hline & KW & 68 & 15 & 15 & 20 & 55 & 15 & 15 & 30 & 65 & -0.09 \\
\hline & TT & 61 & 5 & 5 & 0 & 20 & 5 & 5 & 0 & 5 & -1.93 \\
\hline & EG & 60 & 15 & 20 & 25 & 45 & 15 & 20 & 35 & 60 & 0.04 \\
\hline & GP & 78 & 40 & 40 & 45 & 70 & 35 & 40 & 50 & 70 & 1.89 \\
\hline & DR & 71 & 20 & 20 & 40 & 75 & 30 & 15 & 30 & 80 & 0.67 \\
\hline \multirow[t]{3}{*}{$t$ tests } & Mean & 70.7 & 20.0 & 17.7 & 27.7 & 49.1 & 22.7 & 17.3 & 27.3 & 54.5 & 0.04 \\
\hline & $t(20)$ & 0.17 & 0.00 & 0.41 & 0.27 & 0.6 & 1.2 & 0.52 & 0.86 & 0.61 & 0.20 \\
\hline & $p$ Value & 0.87 & 1.00 & 0.69 & 0.79 & 0.56 & 0.25 & 0.61 & 0.95 & 0.30 & 0.85 \\
\hline
\end{tabular}

N.B.: an overall hearing factor was produced from all pure tone thresholds for each ear using a principal component analysis.

the experiment. Control and WA participants did not show significantly different hearing thresholds at any frequency in the left or right ear (Table 2). An overall hearing score was derived using a principal component analysis. All thresholds for the left and right ear, for each participant were placed into the analysis which produced one overall score (Table 2). There was no difference between the two groups for the overall hearing measure.

\subsection{Assessing acoustic-phonological perception}

Auditory discrimination assessments have been the standard measure of ability in acoustic-phonological perception (e.g. Blumstein et al., 1977; Gainotti et al., 1982; Gow \& Caplan, 1996) in both the clinic and research. These assessments require the participant to judge whether two spoken words or nonwords are phonologically identical (i.e. whether the two words/nonwords sound the same or different to them). The non-identical pairs are most commonly made to differ by one phonemic feature (minimal pairs: e.g. /pat/vs./bat/or/lop/vs./nop/). Nonwords have sometimes been favoured in research studies as they reduce (but probably not do not eliminate) the degree to which the semantic system can support the perceptual system (Franklin, Howard, \& Patterson, 1994; Howard \& Franklin, 1988). All of the participants undertook a standardised nonword minimal pair auditory discrimination assessment from the Psycholinguistic Assessment of Language Processing in Aphasia (PALPA: Kay, Coltheart, \& Lesser, 1992). The results are presented in Table 3.

The results highlight the problems of using a 'same-different' auditory discrimination assessment with the WA population. Firstly, only two of the ten participants (RD and EL) performed significantly above chance. Although this confirms poor performance on this phonological discrimination task, the floor effect means that any underlying systematic variation within the group is missed and it becomes impossible to relate discrimination performance to any measure of comprehension. Varney (1984) noted that phonological discrimination assessments that only utilise select phonological contrasts run the risk of overestimating impairments and that this could be overcome by including more gross phonological contrasts. Secondly, the results show a strong group bias towards responding "same" (the mean accuracy for "same" judgments was 70.3\% compared to $37.8 \%$ for different judgments). Such high rates of false positives have been noted in previous studies of aphasia, including WA (Marshall, Robson, Pring, \& Chiat, 1998; Marshall, 2006). In order to address these problems, a new test of phonological discrimination was developed which utilised an adaptive threshold technique. The new assessment eliminated response bias and

Table 3

WA participants' nonword minimal pair discrimination performance (PALPA 1).

\begin{tabular}{llll} 
& Max score & 72 & \\
Normal cut-off & 65 & Binomial $p$ value \\
& Mean (sd) & $70.8(2.9)$ & \\
\hline $\begin{array}{l}\text { Wernicke's aphasia } \\
\text { participants }\end{array}$ & DL & $\mathbf{3 4}$ & $\mathbf{0 . 6 4}$ \\
& & \\
& LB & 39 & $\mathbf{0 . 2 0}$ \\
CB & 35 & $\mathbf{0 . 5 5}$ \\
MR & 33 & $\mathbf{0 . 7 2}$ \\
RD & 44 & 0.02 \\
DH & $\mathbf{4 2}$ & $\mathbf{0 . 0 6}$ \\
DM & $\mathbf{4 2}$ & $\mathbf{0 . 0 6}$ \\
EL & 45 & 0.01 \\
AC & $\mathbf{4 0}$ & $\mathbf{0 . 1 4}$ \\
CH & $\mathbf{3 7}$ & $\mathbf{0 . 3 6}$ \\
CW & $\mathbf{3 2}$ & $\mathbf{0 . 8 0}$ \\
& & \\
Mean (s.d.) & $38.45(4.55)$ & \\
\% Same correct & 70.3 & \\
\% Different correct & 37.8 &
\end{tabular}

NB: participants are ordered by their severity of auditory comprehension as measured by the BDAE (Goodglass et al., 2001a,b). PALPA = Psycholinguistic Assessment of Language Processing in Aphasia (Kay et al., 1992).

Bold Italicised figures indicate scores that fall within the confidence interval of chance performance on this test. 
Table 4

Levels of phonological confusability within the adaptive staircase discrimination task and example stimuli.

\begin{tabular}{|c|c|c|c|c|}
\hline \multirow[t]{2}{*}{ Level of confusability } & \multicolumn{2}{|c|}{ Confusability of the change to the: } & \multicolumn{2}{|c|}{ Example stimuli } \\
\hline & Onset consonant & Offset consonant & Reference & Target \\
\hline 14 (easiest) & $0.5-5 \%$ & $0.5-5 \%$ & fom & pon \\
\hline 13 & $6-11 \%$ & $0.5-5 \%$ & $b a b$ & vam \\
\hline 12 & $6-11 \%$ & $6-11 \%$ & beeg & veez \\
\hline 11 & $12-20 \%$ & $0.5-5 \%$ & teef & keeb \\
\hline 10 & $12-20 \%$ & $6-11 \%$ & thod & soz \\
\hline 9 & $12-20 \%$ & $12-20 \%$ & tid & kiz \\
\hline 8 & $\geq 21 \%$ & $0.5-5 \%$ & teit & peik \\
\hline 7 & $\geq 21 \%$ & $6-11 \%$ & kav & $p a b$ \\
\hline 6 & $\geq 21 \%$ & $12-20 \%$ & keit & peip \\
\hline 5 & $\geq 21 \%$ & $\geq 21 \%$ & koiv & poith \\
\hline 4 & Unchanged & $0.5-5 \%$ & darf & darp \\
\hline 3 & Unchanged & $6-11 \%$ & berv & berb \\
\hline 2 & Unchanged & $12-20 \%$ & boit & boip \\
\hline 1 (hardest) & Unchanged & $\geq 21 \%$ & nuk & nup \\
\hline
\end{tabular}

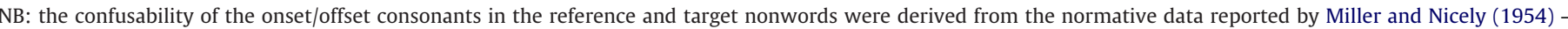

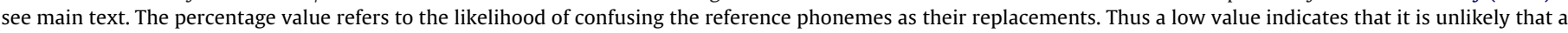

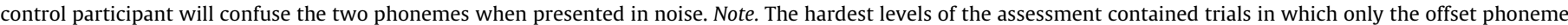
was altered thus minimising the critical acoustic-phonological difference even further.

was much more sensitive to variation in the degree of phonological discrimination skills across the WA case-series.

\subsection{Adaptive maximal pair discrimination assessment}

This assessment used a three-down, one-up adaptive staircase design whereby the degree of difficulty of each trial was adapted online based on the previous response (Cornsweet, 1962). The trials were three-interval, two-alternative forced choice (AXB). The participants were instructed that they would hear three nonwords and to identify which one was different (the odd-one-out). The target was never in the middle. If the target (odd-one-out) was in position $A$ then $X$ and $B$ were the same reference stimulus; if the target was in position $B$ then $A$ and $X$ were the same reference stimulus. The staircase was created by varying the perceptual distance (measured by the degree of phoneme confusability, see Stimuli Creation below) between the reference words and the target word. A total of 14 levels were generated (see Table 4). Level 14 was the easiest (the level with the greatest perceptual distance or least phoneme confusability between the reference and the target) and Level 1 was the hardest (the level with the smallest perceptual distance or most phoneme confusability between the reference and the target). All participants began the assessment at Level 14. The assessment moved down a level (i.e. became harder as the perceptual distance reduced) after three sequential correct responses at the same level and the assessment moved up a level (i.e. made easier as the perceptual distance increased), also termed a reversal, after one incorrect response. The assessment was terminated after one of three circumstances: (a) eight level reversals, indicating the perceptual threshold had been reached; (b) eight incorrect responses at Level 14, indicating inability to achieve the task at the greatest perceptual distance; (c) eight sequential correct responses at Level 1 , indicating ability to discriminate even at the closest perceptual distance. An individual discrimination threshold was calculated for each participant by averaging the levels at which the final four reversals occurred. If the respondent remained at Level 14 or reached and remained at Level 1, then these values were taken as the threshold. Prior to testing, all participants were trained on Level 14 contrasts in order to familiarise them with the task and to set a comfortable volume level.

\subsection{Perceptual distance derivation:}

The perceptual distance for the stimuli for this assessment was derived from Miller and Nicely's (1954) phoneme confusability measures. These measures were acquired by presenting 16 frequent English phonemes in CV syllables [phoneme +/a:/] to listeners at different signal to noise ratios. Their listeners attempted to identify the consonants. Miller and Nicely (1954) recorded the number of times the phonemes were correctly identified and the number and types of phonemes when the target was misidentified. Although the current study presented stimuli without noise, data from the equal signal:noise were selected from the Miller and Nicely (1954) set, due to the high accuracy of phoneme identification at higher signal:noise. 'Confusability rates' were calculated as the percentage of times one phoneme was misidentified as another phoneme for each phoneme pair. For example a/p/was misidentified as a/t/in $12.8 \%$ of presentations. Table 5 sets out the confusability rates derived from this method.

This is not the first test of phonological discrimination to grade stimuli by the degree of difficultly. Morris, Franklin, Ellis, Turner, and Bailey (1996) developed a maximal pairs CVC same-different discrimination test whereby consonant distinctive features (voicing, manner and place of articulation) and vowels were systematically changed to vary the difficulty of judgment. This created trials with a maximal difference where both consonants were changed by three distinctive features as well as a vowel change and a minimum difference when only one distinctive feature of one consonant was changed. This intuitive approach was considered for this study but not selected for three reasons: (1) Distinctive feature contrasts are linguistic in nature rather than perceptual. Although it is known that two distinctive features are more easily discriminated then one distinctive feature (e.g. Morris et al., 1996) it is also clear that not all distinctive features are equally easy or difficult to discriminate; for example, manner is considerably easier to discriminate than place or voicing features (Gow \& Caplan, 1996). (2) Whilst data from previous studies of auditory discrimination show that there is a broad hierarchy of discriminability, vowels $>$ manner and sonorance $>$ place and voicing, there is insufficient evidence to exploit this in test construction, e.g. there is discrepancy of evidence about whether place or voicing contrasts are easier to discriminate (Gow \& Caplan, 1996). (3) The distinctive feature approach only permits a maximum of nine levels. The test developed for this study had 14 levels in an aim to increase sensitivity. However, the number of levels in the current test is dictated by the number of 'confusability bins' selected (see below). This, by its nature, is somewhat arbitrary. However for the current study four confusability bins were chosen to get a balance between sensitivity and practicality. 
Phoneme confusability rates derived from Miller and Nicely (1954).

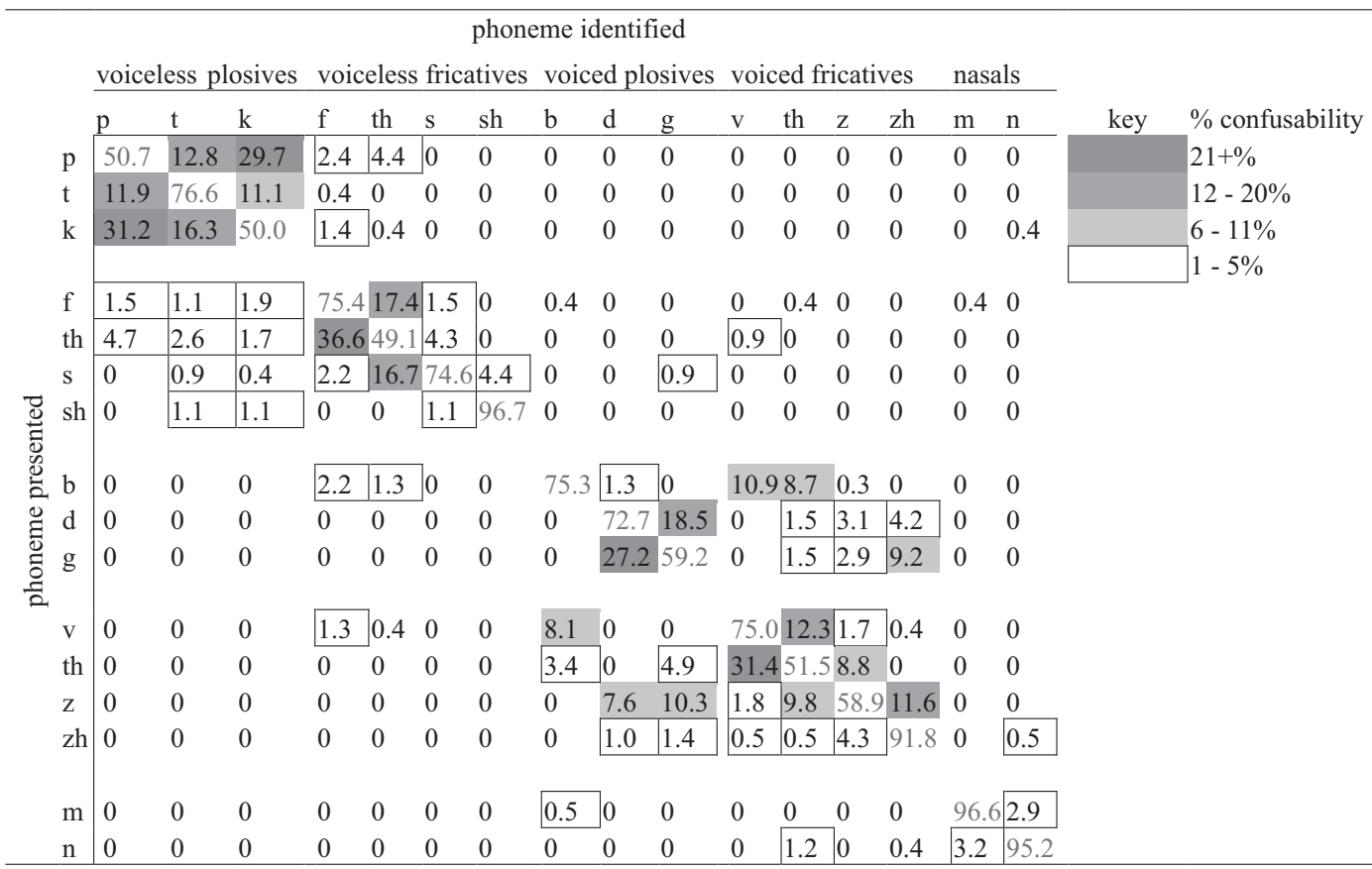

NB: voiceless/th/as in '/th/rough', voiceless/sh/as in '/sh/oe', voiced/th/as in '/th/at', voiced/zh/as in middle sound in mea/s/ure.

\subsection{Stimuli and level creation}

Table 4 presents example stimuli for each test level, full stimuli details are available in the online supplementary materials. All 700 stimuli were one syllable CVC strings. The vowel did not change within a trial but did change between trials and included both long and short vowels. The 14 test levels were created by separating the confusability rates into four bins: $\geq 21 \%, 12-20 \%, 6-11 \%$ and $0.5-5 \%$ confusability. The $\geq 21 \%$ had the most confusable phoneme pairs and contained misidentifications made on $\geq 21 \%$ of presentations; e.g. a/p/was identified as a/k/on $29.7 \%$ of presentations. The $0.5-5 \%$ bin had least confusable phoneme pairs and contained misidentifications made on $0.5-5 \%$ of trials, e.g. a/p/was identified as a/f/on only $2.4 \%$ of trials. Phonemes pairs confused less than $0.5 \%$ of the time were not included in the experiment.

These confusability levels were then systematically paired (see Table 4) to create the 14 test levels. The pairing process involved not just the selection of which phoneme to be substituted but also in which position they should be substituted (i.e. both initial and final or final only). At the easier staircase levels (Levels 14-5), both the initial and final phonemes of the target were changed compared to the reference nonword (the stimulus in position $\mathrm{X}$ ). At the harder levels (Levels 4-1), only the final, less salient (Burns \& Canter, 1977) phoneme was changed in the target compared to the reference nonword. Miller and Nicely's (1954) data only provide measures of phoneme confusability in initial positions. However, as CVC stimuli were used in the current test, the same degrees of confusability were taken for phonemes in final position. A coarse assumption was taken that syllable final phonemes would be perceptually more difficult to distinguish as this has been observed previously in both control and aphasia participants (Gow \& Caplan, 1996).

To make the stimuli, reference nonwords were selected and then the phonemes from that reference item were systematically replaced by phonemes from the appropriate confusability bin (as summarised in Table 4). For example, on Level 14 (easiest) both the initial and final phonemes were replaced by phonemes in the $0.5-5 \%$ confusability bin (least confusable); e.g. reference $=/$ mouk $/$ target $=/$ nouth $/$. For Level 13 the initial phoneme was replaced by a phoneme from the $6-11 \%$ confusability bin and the final phoneme was replaced by a phoneme from the $0.5-5 \%$ confusability bin; e.g. reference $=/$ dees/, target $=/$ zeef/and so on. For Level 1 (hardest) only the final phoneme was replaced by a phoneme from the $21+\%$ confusability bin (most confusable); e.g. reference $=/ \mathrm{kiv} /$, target $=/$ kith $/$.

\subsection{Test construction}

Seven hundred stimuli were created and recorded in a female voice with a Thames Estuary accent on a Tascam DA-P1 digital audio tape recorder at a sample rate of $44.1 \mathrm{kHz}$. These were used to generate 25 trials per level. For each stimulus the pitch contour was manipulated so all stimuli had an equal, slightly falling intonation. This was implemented using the PSOLA algorithm in the Praat software (Boersma \& Weenink, 2009). Stimulus duration was not manipulated as vowel duration and other temporal cues are important features of phoneme identification (Raphael, 1972; Shannon, Zeng, Kamath, Wygonski, \& Ekelid, 1995). However, the whole trial length was kept constant by placing the stimuli in $750 \mathrm{~ms}$ time blocks separated by a $500 \mathrm{~ms}$ ISI. This produced a total trial length of $3250 \mathrm{~ms}$.

\subsection{Administration}

The test was programmed in E-Prime 1.1 (Psychology Software Tools) and presented over Sennheiser HD 380 pro headphones via a cakewalk UA-4FX sound card. Participants adjusted the volume via the sound card to a comfortable level following instruction by the experimenter. 
Table 6

Adaptive staircase discrimination thresholds for WA and control participants.

\begin{tabular}{llll}
\hline WA participant & Threshold & Matched control participant & Threshold \\
\hline DL & 10.5 & DW & 1 \\
LB & 13 & PB & 1 \\
CB & 13 & PD & 1 \\
MR & 11 & HW & 1 \\
RD & 9.5 & TA & 1 \\
DH & 11.5 & HE & 1 \\
DM & 8.5 & KW & 1 \\
EL & 2 & TT & 1 \\
AC & 2 & EG & 1 \\
CH & 3.5 & GP & 1 \\
CW & 1 & DR & 1.5 \\
Mean & & & 1.05 \\
s.d. & 7.77 & & 0.15 \\
\hline
\end{tabular}

NB: discrimination threshold reflects the confusability level (see Table 4 and main text) at which the participants were able to detect a difference.

\section{Results}

\subsection{Control participants}

Maximal pair discrimination thresholds for the WA and control participants are presented in Table 6 . All the control participants except one (DR) were able to achieve a discrimination threshold at the most difficult level even though their staircases were not error-free per se.

\subsection{Wernicke's aphasia participants}

The results from the new discrimination test confirm a severe acoustic-phonological impairment in individuals with WA. All WA participants produced appropriate staircases (Fig. 3). This indicated that they were able to achieve the task requirements, as it is unlikely that a staircase shape would be produced by chance (i.e. guessing), and therefore participants were able to generate a reliable threshold. In addition, the task was able to differentiate a range of discrimination thresholds across the WA participants. At a group level, the WA participants were significantly worse than age and hearing matched control participants $\left(t_{(20)}=4.80, p<0.001\right)$. This indicated that lower levels of phoneme confusability (between reference and target) were required before the WA participants could reliably discriminate between the nonwords. The majority of the WA group (7/11: DL, LB, CB, MR, RD, DH, DM) had a threshold at high levels (14-5) at which point both the initial and final phonemes differed between the reference and the target. Two of the three most severe participants were only able to discriminate at Levels 14 and 13 - where both the initial and final phoneme were changed to phonemes with low levels of confusability. Those participants with comprehension scores above the 15th centile on the BDAE (Goodglass et al., 2001b) were able to discriminate nonwords where only the final phoneme changed and 3 out of 4 of these participants were able to do this at high levels of confusability (12-20\% - Level 2). Only one WA participant ( $\mathrm{CW}$ ) performed at the same level as the controls. This participant had the mildest comprehension impairment, as indicated by his BDAE profile (see Fig. 1), though he was not the strongest performer on the minimal pair test (Table 3).

\subsection{Relationship between phoneme discrimination thresholds and auditory comprehension}

To determine the strength of association between the degree of impairment in phonological discrimination and auditory comprehension, a Spearman's correlation was computed between threshold level and combined auditory comprehension scores from the BDAE (which included spoken word picture matching, commands and complex ideational material; see Table 1 for BDAE scores). A significant relationship between auditory discrimination thresholds and auditory comprehension scores overall (Spearman's rho $=-.83, p=0.002$ ) was found (Fig. 4). Spoken word to picture matching, following commands and responding to questions ('complex ideational material') provide different types of contextual information. The former provides visual information and only requires acoustic-phonological processing of single words, the later two provide no visual information but wider sentence level linguistic information and require a greater degree of acoustic-phonological processing. To investigate whether a relationship between auditory discrimination and auditory comprehension with these different degrees of contextual information occurs, further correlations were undertaken. Significant correlations were found between auditory discrimination and spoken word to picture matching (Spearman's rho $=-0.68, p=0.022$ ) as well as sentence level comprehension (commands and complex ideational material) (Spearman's rho $=-0.72, p=0.013$; Fig. 4 ).

\subsection{Relationship between nonverbal semantic skills and auditory comprehension}

To establish whether there was a relationship between impaired nonverbal semantic skill, (measured using the 3 picture version of Pyramids and Palm Test (PPT: Howard \& Patterson, 1992) and auditory comprehension (overall BDAE score) a second correlation was carried out. No significant relationship was found (Spearman's rho $=0.52, p=0.1$ )

\subsection{Predicting auditory comprehension in WA}

To establish how well auditory comprehension scores could be predicted for all the WA participants by the new phonological discrimination task (adaptive staircase task) and the nonverbal semantic assessment (PPT task), both factors were entered into a multiple regression with auditory comprehension as the dependent variable. This produced a significant model $\left(F_{(2)}=7.4, p=0.015\right)$. Auditory discrimination made a significant contribution to the model $(t=3.1, p=0.015)$ whilst PPT scores made no significant contribution $(t=0.27, p=0.79)$.

\subsection{Relationship between hearing, auditory discrimination and auditory comprehension}

The majority of the participants in the study showed a degree of age related hearing loss, albeit to different extents. The control participants effectively achieved the auditory discrimination task, despite a similar degree of hearing impairment to the WA participants. The one control participant who did not have a discrimination threshold at ceiling (DW), did not show the greatest degree of hearing loss. In the WA group, the only participant to reach the level of the control participants (CW) had the greatest degree of hearing loss. To confirm that hearing loss did not play a significant role in the auditory discrimination or auditory comprehension abilities in the WA group, correlations between the overall hearing factor for each participant (produced using a principal component analysis, see Section 2.4) and auditory discrimination threshold and auditory comprehension scores were performed (Fig. 5). No significant correlations between hearing and any other skill were observed (hearing and discrimination: Spearman's rho $=-0.57, p=0.067$, hearing and total comprehension: Spearman's rho $=0.45, p=0.17$, hearing and sWPM: Spearman's rho $=0.58, p=0.064$, hearing and sentence comprehension: Spearman's rho $=0.32, p=0.34$ ). 


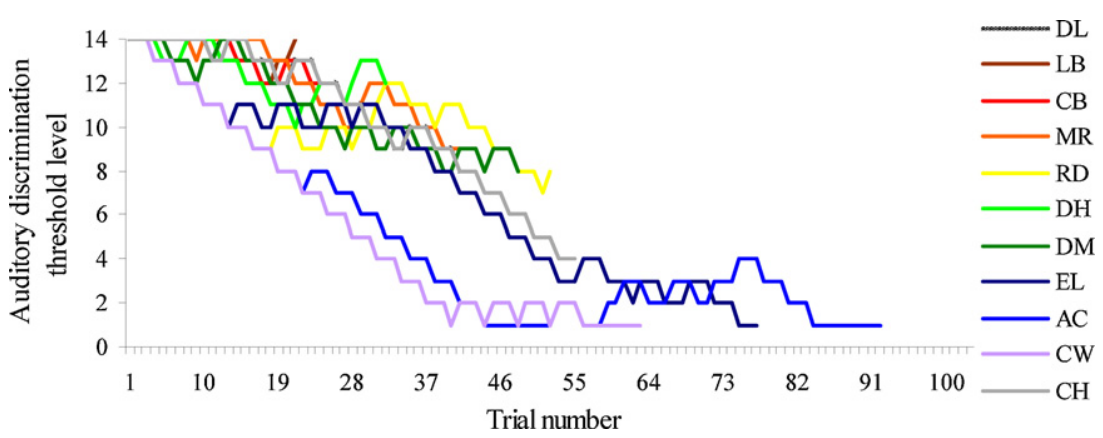

Fig. 3. Auditory discrimination staircase results for Wernicke's aphasia participants.
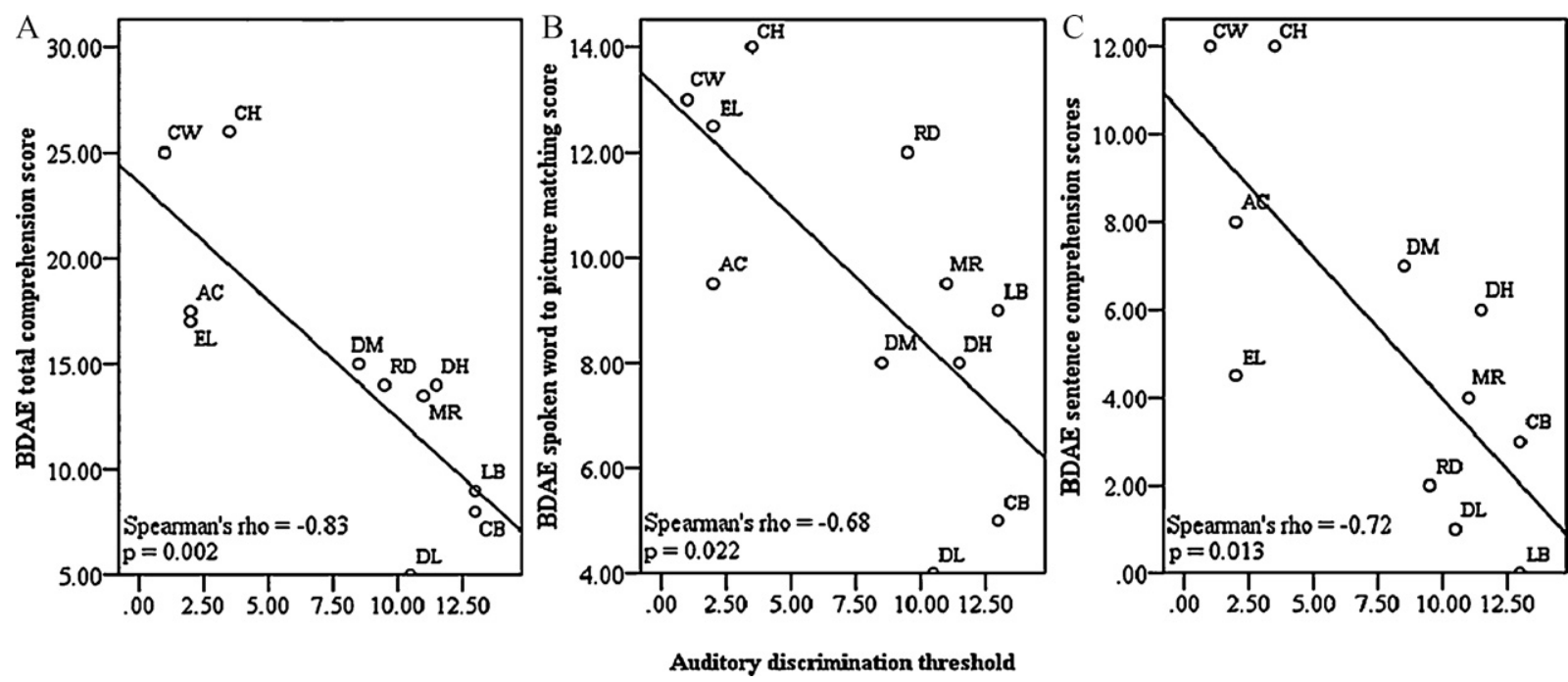

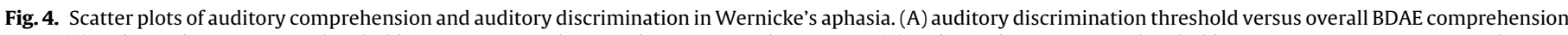

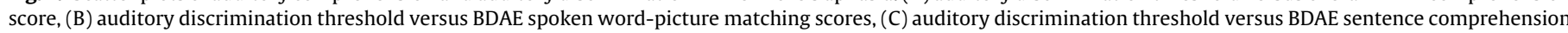
scores.
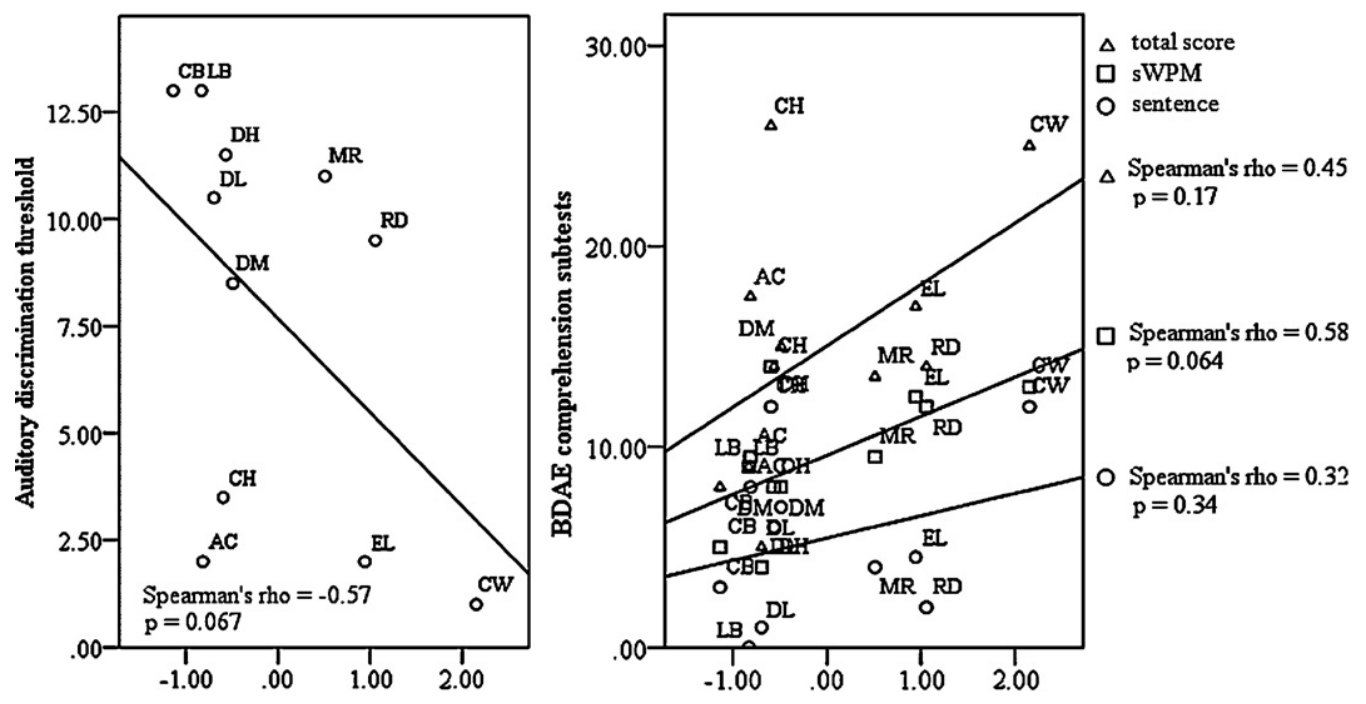

Hearing factor

Fig. 5. Scatter plots showing relationship between overall hearing factor and auditory discrimination thresholds and comprehension scores in Wernicke's aphasia.

\section{Discussion}

This study found evidence that impaired phonological analysis underlies the comprehension impairment in WA. Participants with
WA were severely impaired at auditory phonological discrimination compared to age and hearing matched controls and the degree of this impairment correlated with auditory comprehension both at a single word and sentence level. This result is consistent with 
traditional hypotheses such as Luria's (1976) ‘decoding deficit'. The decoding deficit hypothesis predicts that impaired phonological analysis of the speech stream will be responsible for impaired comprehension, particularly under formal testing conditions. In comparison, intact prosodic analysis of the speech stream leads to apparently better comprehension in contextual/social situations. Previous attempts to find behavioural evidence for Luria's decoding deficit have not provided significant results (Blumstein et al., 1977; Gainotti et al., 1982). Because no significant correlation was found between auditory discrimination scores and auditory comprehension scores, Blumstein et al. (1977) were not able to find evidence that the comprehension impairment in WA could be accounted for by a single underlying impairment (or selective impairment) to phonological perception and instead suggested this impairment played only a contributory role. The lack of evidence for such a relationship has given more prominence to role of semantics as the main driver of comprehension problems in WA (Gainotti et al., 1982; Hickok, 2000). However, no positive evidence for a link between either underlying cognitive skill and comprehension behaviours has previously been established. The current study differs from Blumstein et al. (1977) in two key ways: the number of participants studied and the method of testing auditory analysis skills. By increasing the size of the WA case-series and using a test which not only detected the presence of the WA participants' phonological decoding deficit but also was sensitive to the degree of impairment, a significant association was shown between phonological-discrimination and verbal comprehension scores. In short, this study found clear and strong evidence for the elusive link between phonological analysis and the auditory comprehension deficit in WA.

The nature of the new assessment was critical in being able to demonstrate this link, particularly in its ability to overcome the problems associated with the traditional 'same-different' minimal pair discrimination tasks. On such assessments, the current WA participants - like those reported previously - performed at floor levels and produced a large proportion of false positive responses. As such, whilst this type of task demonstrates the presences of a prominent phonological impairment in WA, it prevents any attempt to relate the degree of impairment to the comprehension performance and, additionally, it is not possibly to confirm task comprehension by participants. Instead, a highly graded auditory discrimination test was devised by utilising information about phonological confusability (Miller \& Nicely, 1954). This allowed the perceptual distance between the target and reference stimuli to be varied systematically and adaptively. An adaptive approach is particularly useful in clinical populations as it allows for confirmation of success in carrying out task requirements and speed in obtaining reliable thresholds for each participant by eliminating repeated exposure to stimuli at inaccessible levels. This measure has parallels with a previous phonological discrimination measure developed by Morris et al. (1996) which linguistically controlled the distance between the discrimination stimuli by manipulating the distinctive features of place, manner and voicing. Whilst there is high overlap between the distinctive feature approach and the perceptual approach there is not total agreement. For example, the distinctive feature approach makes no distinction in difficulty between $/ \mathrm{p} / \mathrm{vs}$./t/ and /p/vs./k/, but based on the perceptual approach these distinctions belonged to two different confusability brackets. This is, however, somewhat arbitrary based on what confusability bands are chosen. Overall, it is likely that the linguistic and perceptual approaches would produce similar results.

Given that verbal comprehension impairment and fluent (in some cases jargonistic) output are the critical diagnostic features of WA and the features resistant to therapeutic change, then the demonstration that this can be predicted by the participants' degree of phonological analysis problems is a significant step forward. This does not mean, however, that this deficit is sufficient to explain all of the participants' impairments but rather that it is the major factor in their poor verbal comprehension. Previous studies (Cohen et al., 1980; De Renzi et al., 1972; Gainotti et al., 1983; Robson et al., in press), have shown that some participants with WA also present with nonverbal comprehension impairments. Indeed, this has been used in the past to argue against the phonological analysis hypothesis. Such debates are predicated on the assumption that there is a single underlying (selective) deficit in WA. Instead, it appears more likely that there are at least two independent factors that become related through the proximity of the critical underlying neural regions. Previous studies have shown that verbal comprehension impairment following posterior MCA stroke tends to be associated with a lesion overlap in PSTG and primary auditory cortex (Chertkow, Bub, Deaudon, \& Whitehead, 1997; Robson et al., in press). This is joined by nonverbal comprehension deficits when the lesion extends into pMTG and AG regions (Chertkow et al., 1997). It is common for lesions in WA to extend into these nonverbal areas (see lesion overlap in this study and Dronkers, Redfern, \& Ludy, 1995; Dronkers et al., 2004; Ogar et al., 2011). Although the correlation in the current study between nonverbal semantics, measured by the Pyramids and Palm Tree test (PPT), and auditory comprehension did not quite reach significance it is unlikely that an impairment to a nonverbal semantic system plays no role in the comprehension impairment in patients with WA. The semantic and phonological systems are highly interactive (Plaut \& Shallice, 1993). The effects of this interactivity can be witnessed in other neuropsychological conditions including in "deep" disorders, whereby weaknesses in the phonological system are compensated for by the relative strength of the semantic system (Jefferies, Sage, \& Lambon Ralph, 2007). This interactivity means that, in the case of WA, small degrees of disruption to the semantic system may have a disproportionate impact on comprehension because (a) underspecified or distorted phonological input will be making contact with a weakened semantic system and (b) a weak semantic system will have reduced capacity to support the phonological system through interactive processes. The reciprocal effects of the semantic and phonological systems have been experimentally observed in WA. Baker et al. (1981) showed that the degree of phonological complexity increased the number of semantic errors on word to picture matching and, likewise, the degree of semantic complexity impacted performance on phonological discrimination. Additionally, it is possible that the lack of significant correlation between non-verbal semantic analysis and auditory comprehension was due to insensitivity in the testing materials. The PPT (non-verbal semantic assessment) is a useful clinical tool to identify the presence of a semantic impairment. However, as the semantic distance in the PPT is not manipulated in the systematic way that the phonological perceptual distance was manipulated in the adaptive task developed for the current study, it is possible that there may be an underestimation in the degree of relationship between auditory comprehension and semantic processing in the current group. At present, these results appear consistent with the interpretation that impaired acoustic-phonological analysis plays a more primary role, and impaired semantic processing plays a contributory role in WA (Robson et al., in press). In this study 5/11 participants were within normal limits on the PPT whereas only one participant performed at a similar level to the control participants on auditory discrimination. However, more sensitive assessment of nonverbal semantic processing is required to confirm whether a contribution of impaired semantic processing can be seen throughout WA or only at the individual/sub-group level.

This study has been able to elucidate one broad cognitive pattern underlying WA. Whilst this is clinically important, individual variation must not be overlooked. For example, whilst the WA group were homogeneous in respect to their overall behavioural 
characteristics, individuals displayed severity variation on various assessments. The strength of the relationship between phonological discrimination and comprehension should not detract from the need to establish the integrity of a range of skills in individuals with WA, particularly semantic abilities and, potentially, non-verbal auditory skills. This multi-dimensional approach will more comprehensively account for the comprehension deficit in WA.

Thus, in many cases, the comprehension impairment in WA cannot be accounted for by a single selective deficit in acoustic-phonological analysis. It is also worth noting that impaired acoustic-phonological deficits do not affect individuals with WA selectively. More specifically, impairments on phonological discrimination and phonological identification tasks have been observed in most subtypes of aphasia. Correlations between phonological discrimination and auditory comprehension have been identified previously over groups of aphasia participants with a range of clinical diagnoses (Blumstein et al., 1977; Miceli et al., 1980; Tallal \& Newcombe, 1978). However, these correlations are inconsistent with other studies failing to find any relationship (e.g. Basso et al., 1977; Jauhiainen \& Nuutila, 1977). Such studies which include multiple aphasia subtypes are difficult to interpret. Considering the inevitable variety in lesion distribution and residual functional language networks included, it is perhaps unsurprising that a lack of consistent correlations between acoustic-phonological processing skills and comprehension impairments have been established.

It is probable that surface behavioural impairments in acoustic-phonological processing tasks such as phonological discrimination have different underlying causes and therefore different consequences for linguistic processing. As well as disrupted acoustic-phonological analysis per se, impairments on phonological discrimination tasks could arise from general processing resource limitations or working memory impairments (Burton, Small, \& Blumstein, 2000; Gow \& Caplan, 1996). Observed correlations may be a product of an artificial relationship where some subtypes of aphasia show less severe profiles overall. Blumstein et al. (1977) comment that the correlation found in the aphasia group in their study was driven by superior performance in Broca's aphasia, rather than systematic changes on an individual-by-individual basis. Whilst correlations between acoustic-phonological analysis and auditory comprehension in aphasia as a whole may be spurious, it may be reasonable to attempt to find such correlations with homogeneous subtypes. Wernicke's aphasia presents the most likely candidate to show a direct link between acoustic-phonological analysis itself and auditory comprehension due to the proximity of the lesion to primary and secondary auditory cortices (Bogen \& Bogen, 1976). Further work is required to establish (a) whether individuals with different subtypes of aphasia but overlapping lesion profiles show a similar relationship between acoustic-phonological analysis and auditory comprehension and (b) whether individuals with different aphasia subtypes and lesion profiles (including frontal varieties) show a relationship between scores on acoustic-phonological tests and assessments proposed to index the behaviours that have been related to those neural regions (e.g. working memory in frontal areas: Burton et al., 2000).

The results from the current study are consistent with the traditional account of Wernicke's aphasia (Eggert, 1977). However, the dual stream model of language (Hickok \& Poeppel, 2004, 2007) proposes that phonological analysis for auditory comprehension is undertaken bilaterally in the superior temporal plane and that the comprehension impairment in WA is a result of impaired lexicalsemantic integration post intact acoustic-phonological analysis (Hickok, 2000). The null results from Blumstein et al. (1977) and Gainotti et al. (1982) have been taken to be consistent with this alternative hypothesis. The results from this study, however, appears contradictory to this view in that the primary impairment underlying the verbal comprehension impairment was acoustic-phonological and this deficit arose in the context of a left hemisphere unilateral lesion for all the individuals in this study. The strongest support for bilateral involvement in phonological processing comes from fMRI and TMS explorations in neurologicalintact participants (e.g. Binder et al., 2000; Hartwigsen et al., 2010). Studies of receptive language processing are associated with bilateral STG activation (Narain et al., 2003; Scott, Blank, Rosen, \& Wise, 2000); Vigneau et al. (2011) have argued that right hemisphere activations might reflect more general acoustic processing or other aspects of language stimuli (e.g. prosody, etc.) When more complex, non-language contrast stimuli have been used in functional neuroimaging studies of speech processing then the resultant activation patterns have been much more left-lateralised in form (Narain et al., 2003; Scott et al., 2000). The mechanisms behind the emergence of this left lateralisation are not clear. One theory has proposed that asymmetries emerge due to the nature of representations that acoustic information must interface with, i.e. lexicosemantic information in the left hemisphere. A second approach has proposed asymmetries in neural acoustic sampling rate might lead to a left hemisphere advantage for phonological processing due to a left hemisphere preference for analysis of fast temporal rates over which phonological information is conveyed (Boemio, Fromm, Braun, \& Poeppel, 2005). Wernicke's aphasia may provide an interesting group within which to study these hypotheses.

In this regard, it is important to note that not all lesions to the superior temporal lobe and temporoparietal junction result in WA (Dronkers et al., 2004). Individuals with lesions to these areas sometimes resolve to conduction or anomic aphasias at the chronic stage (Pedersen, Vinter, \& Olsen, 2004). This phenomenon may be accounted for by the rapid plasticity observed in the auditory system (Ohl \& Scheich, 2005). The human auditory cortices show changes in BOLD signal in response to auditory stimuli preand post-training and animal electrophysiology studies show rapid online plasticity in response to repeated auditory stimuli (Ohl \& Scheich, 2005). For example, in an fMRI study Mottonen et al. (2006) showed auditory cortex changes in response to sine wave speech after training, indicating recruitment of wider areas within the auditory network in the left hemisphere following training which, in turn, heightened awareness of the intelligibility of stimuli. Aphasia participants with lesions to the left auditory association cortex show greater recruitment of right hemisphere auditory association cortex in response to increased speech rate compared to unimpaired participants (Leff et al., 2002). Thus the primary and associative auditory cortices may be particularly amenable to reorganisation (e.g. Saygin, Leech, \& Dick, 2010). Those who are unable to undergo this kind of functional reorganisation may stay within the WA category in the chronic phase. This raises important questions about the differences between those who recover and those who do not. Factors may include age (Keidel, Welbourne, \& Lambon Ralph, 2010), precise lesion locations (Price, Seghier, \& Leff, 2010) including patterns of white-matter disconnection, availability of other 'higher level' neural resources to support recovery (Jaaskelainen, Ahveninen, Belliveau, Raij, \& Sams, 2007) or disruption to lower level perceptual inputs into the phonological system. Further light may come from longitudinal, prospective examination of individuals with WA, alongside understanding underlying factors in chronic WA.

\section{Conclusions}

This study was able to show a link between auditory comprehension and Wernicke's aphasia that had, in previous studies (Blumstein et al., 1977; Gainotti et al., 1982) been elusive. The positive result from the current study highlights two important issues in 
cognitive neuropsychology. Firstly the clinical group being studied must be accurately defined using behavioural and, where possible, lesion data. Over inclusion of multiple clinical subtypes who share only one or two behavioural features cannot reveal distinct patterns that may be unique to or underlie particular clinical disorders. Secondly, this study highlights the importance of using graded assessments. Total failure on a task is not informative for observing systematic behavioural patterns within a group. The novel assessment used in the current study was graded and sufficiently sensitive to produce representative variation in the underlying skill (phonological discrimination) that it was attempting to index.

\section{Acknowledgements}

We would like to thank the participants and their relatives/carers for their assistance in this study and the speech and language therapists in the North of England who have referred participants to this project. We would like to thank Yu Li for his expertise in E-Prime script programming. This work was supported by a Stroke Association Allied Health Professional Research Bursary (TSAB2008/01) to Holly Robson and an MRC programme grant to MALR (G0501632 \& MR/J004146/1).

\section{Appendix A. Supplementary data}

Supplementary data associated with this article can be found, in the online version, at doi:10.1016/j.neuropsychologia.2011.11.022.

\section{References}

Ashburner, J., \& Friston, K. J. (2005). Unified segmentation. Neuroimage, 26(3) 839-851.

Baker, E., Blumstein, S. E., \& Goodglass, H. (1981). Interaction between phonologica and semantic factors in auditory comprehension. Neuropsychologia, 19, 1-15.

Basso, A., Casati, G., \& Vignolo, L. A. (1977). Phonemic identification defect in aphasia. Cortex, 13(1), 85-95.

Baum, S. R. (2002). Sensitivity to sub-syllabic constituents in brain-damaged patients: Evidence from word games. Brain and Language, 83(2), 237-248.

Binder, J. R., Frost, J. A., Hammeke, T. A., Bellgowan, P. S. F., Springer, J. A., Kaufman, J. N., et al. (2000). Human temporal lobe activation by speech and nonspeech sounds. Cerebral Cortex, 10(5), 512-528.

Blumstein, S. E., Baker, E., \& Goodglass, H. (1977). Phonological factors in auditory comprehension in aphasia. Neuropsychologia, 15, 19-30.

Boemio, A., Fromm, S., Braun, A., \& Poeppel, D. (2005). Hierarchical and asymmetric temporal sensitivity in human auditory cortices. Nature Neuroscience, $8,389-395$

Boersma, P., \& Weenink, D. (2009). Praat: Doing phonetics by computer (Version 5.1.05). http://praat.org

Bogen, J. E., \& Bogen, G. M. (1976). Wernicke's region - Where is it? Annals of the New York Academy of Sciences, 280, 834-843.

Boller, F., Kim, Y., \& Mack, J. L. (1977). Auditory comprehension in aphasia. In H. W. H. A. Whitaker (Ed.), Studies in Neurolinguistics (pp. 1-63). Academic Press: New York

Burns, M. S., \& Canter, G. J. (1977). Phonemic behavior of aphasic patients with posterior cerebral lesions. Brain and Language, 4, 492-507.

Burton, M. W. Small, S. L., \& Blumstein, S. E. (2000). The role of segmentation in phonological processing: An fMRI investigation. Journal of Cognitive Neuroscience, 12(4), 679-690.

Chertkow, H., Bub, D., Deaudon, C., \& Whitehead, V. (1997). On the status of object concepts in aphasia. Brain and Language, 58, 203-232.

Cohen, R., Kelter, S., \& Woll, G. (1980). Analytical competence and language impairment in aphasia. Brain and Language, 10, 331-351.

Cornsweet, T. N. (1962). Staircase - method in psychophysics. American Journal of Psychology, 75(3), 485-\&

Csepe, V., Osman-Sagi, J., Molnar, M., \& Gosy, M. (2001). Impaired speech perception in aphasic patients: Event-related potential and neuropsychological assessment. Neuropsychologia, 39(11), 1194-1208.

Damasio, H., \& Damasio, A. R. (1989). Lesion Analysis in Neuropsychology. New York: Oxford University Press.

De Renzi, E., Faglioni, P., Scotti, G., \& Spinnler, H. (1972). Impairment in associating colour to form, concomitant with aphasia. Brain, 95(2), 293-304.

Dronkers, N. F., Redfern, B. B., \& Ludy, C. A. (1995). Lesion localization in chronic Wernicke's aphasia. Brain and Language, 51, 62-65.

Dronkers, N. F., Wilkins, D. P., Van Valin, R. D., Jr, Redfern, B. B., \& Jaeger, J. J. (2004) Lesion analysis of the brain areas involved in language comprehension. Cognition, 92, 145-177.
Eggert, G. H. (1977). Wernicke's Works on Aphasia: A Sourcebook and Review. The Hague: Mouton Publishers.

Ellis, A. W., Miller, D., \& Sin, G. (1983). Wernicke's aphasia and normal language processing: A case study in cognitive neuropsychology. Cognition, 15, 111-144.

Franklin, S. E., Howard, D., \& Patterson, K. (1994). Abstract word meanign deafness. Cognitive Neuropsychology, 11, 1-34

Gainotti, G., Miceli, G., Silveri, M. C., \& Villa, G. (1982). Some anatomoclinical aspects of phonemic and semantic comprehension disorders in aphasia. Acta Neurologica Scandinavica, 66(6), 652-665.

Gainotti, G., Silveri, M. C., Villa, G., \& Caltagirone, C. (1983). Drawing objects from memory in aphasia. Brain, 106, 613-622.

Gates, G. A., Cooper, J. C., Kannel, W. B., \& Miller, N. J. (1990). Hearing in the elderly the framingham cohort, 1983-1985. 1. Basic audiometric test - Results. Ear and Hearing, 11, 247-256.

Goodglass, H., \& Kaplan, E. (1972). The Assessment of Aphasia and related disorders. Philadelphia: Lea \& Febiger.

Goodglass, H., Kaplan, E., \& Barresi, B. (2001a). The Assessment of Aphasia and related disorders (3rd ed.). Baltimore: Lippincott Williams \&Wilkins.

Goodglass, H., Kaplan, E., \& Barresi, B. (2001b). Boston Diagnostic Aphasia Examination - 3rd ed., (BDAE). Baltimore: Lippincott Williams \& Wilkins.

Gow, D. W., \& Caplan, D. (1996). An examination of impaired acoustic-phonetic processing in aphasia. Brain and Language, 52(2), 386-407.

Hartwigsen, G., Baumgaertner, A., Price, C. J., Koehnke, M., Ulmer, S., \& Hartwig, R. S. (2010). Phonological decisions require both the left and right supramarginal gyri. Proceedings of the National Academy of Sciences of the United States of America, 107, 16494-16499.

Hickok, G. (2000). Speech perception, conduction aphasia and the functional neuroanatomy of language. In Y. Grodzinsky, L. P. Shapiro, \& D. Swinney (Eds.) Language and the Brain: Representation and processing. San Diego: Academic Press.

Hickok, G., \& Poeppel, D. (2004). Dorsal and ventral streams: A framework for understanding aspects of the functional anatomy of language. Cognition, 92, 67-99.

Hickok, G., \& Poeppel, D. (2007). The cortical organization of speech processing. Nature Reviews: Neuroscience, 8(5), 393-402.

Howard, D., \& Franklin, S. E. (1988). Missing the Meaning. Cambridge, MA: MIT Press. Howard, D., \& Patterson, K. (1992). Pyramids and Palm Trees: A test of semantic access from pictures and words. Bury Saint Edmunds: Thames Valley Test Company.

Jaaskelainen, I. P., Ahveninen, J., Belliveau, J. W., Raij, T., \& Sams, M. (2007). Short-term plasticity in auditory cognition. Trends in Neurosciences, 30(12), 653-661.

Jauhiainen, T., \& Nuutila, A. (1977). Auditory-perception of speech and speech sounds in recent and recovered cases of aphasia. Brain and Language, 4(4), 572-579.

Jefferies, E., Sage, K., \& Lambon Ralph, M. A. (2007). Do deep dyslexia, dysphasia and dysgraphia share a common phonological impairment? Neuropsychologia, 45(7), 1553-1570.

Kay, J., Coltheart, M., \& Lesser, R. (1992). Psycholinguistic Assessments of Language Processing in Aphasia (PALPA). Hove, UK: Laurence Erlbaum Associates.

Keidel, J. L., Welbourne, S. R., \& Lambon Ralph, M. A. (2010). Solving the paradox of the equipotential and modular brain: A neurocomputational model of stroke vs. slow-growing glioma. Neuropsychologia, 48(6), 1716-1724.

Leeper, H. A., Shewan, C. M., \& Booth, J. C. (1986). Altered acoustic cue discrimination in Broca's and conduction aphasia. Journal of Communication Disorders, 19(2), 83-103.

Leff, A., Crinion, J., Scott, S. K., Turkheimer, F., Howard, D., \& Wise, R. J. S. (2002). A physiological change in the homotopic cortex following left posterior temporal lobe infarction. Annals of Neurology, 51, 553-558.

Luria, A. R. (1976). Disturbances of Understanding of Verbal Communication in patients with sensory aphasia. The Hague: Mouton \& Co. B.V.

Marshall, J. (2006). Jargon aphasia: What have we learned? Aphasiology, 20, 387-410.

Marshall, J., Robson, J., Pring, T., \& Chiat, S. (1998). Why does monitoring fail in jargon aphasia? Comprehension, judgment, and therapy evidence. Brain and Language, 63, 79-107.

Miceli, G., Arena, R., Caltagirone, C., Gianotti, G., \& Payer-Rigo, P. (1977). Phoneme discrimination disturbances in unilateral brain damage patients. Acta Neurologica (Naples), 32(4), 399-412.

Miceli, G., Gainotti, G., Caltagirone, C., \& Masullo, C. (1980). Some aspects of phonological impairment in aphasia. Brain and Language, 11(1), 159-169.

Miller, G. A., \& Nicely, P. (1954). An analysis of the confusion among english consonants heard in the presence of random noise. Journal of the Acoustical Society of America, 26(5), 953-953.

Morris, J., Franklin, S., Ellis, A. W., Turner, J. E., \& Bailey, P. J. (1996). Remediating a speech perception deficit in an aphasic patient. Aphasiology, 10(2), 137-158.

Mottonen, R., Calvert, G. A., Jaaskelainen, I. P., Matthews, P. M., Thesen, T., Tuomainen, J., et al. (2006). Perceiving identical sounds as speech or non-speech modulates activity in the left posterior superior temporal sulcus. Neuroimage, 30(2), 563-569.

Narain, C., Scott, S. K., Wise, R. J. S., Rosen, S., Leff, A. P., Iversen, S. D., et al. (2003). Defining a left-lateralized response specific to intelligible speech using fMRI. Cerebral Cortex, 13, 1362-1368.

Ogar, J. M., Baldo, J. V., Wilson, S. M., Brambati, S. M., Miller, B. L., Dronkers, N. F., et al. (2011). Semantic dementia and persisting Wernicke's aphasia: Linguistic and anatomical profiles. Brain and Language, 117(1), 28-33.

Ohl, F. W., \& Scheich, H. (2005). Learning-induced plasticity in animal and human auditory cortex. Current Opinion in Neurobiology, 15(4), 470-477. 
Pedersen, P. M., Vinter, K., \& Olsen, T. S. (2004). Aphasia after stroke: Type, severity and prognosis - The Copenhagen aphasia study. Cerebrovascular Diseases, 17, 35-43.

Plaut, D. C., \& Shallice, T. (1993). Deep dyslexia - A case study of connectionist neuropsychology. Cognitive Neuropsychology, 10(5), 377-500.

Price, C. J., Seghier, M. L., \& Leff, A. P. (2010). Predicting language outcome and recovery after stroke: The PLORAS system. Nature Reviews Neurology, 6(4), 202-210.

Raphael, L. J. (1972). Preceding vowel duration as a cue to perception of voicing characteristic of world-final consonants in american english. Journal of the Acoustical Society of America, 51(4), 1296-\&.

Robson, H., Sage, K., \& Lambon Ralph, M.A. Wernicke's apahsia reflects a combination of acoustic-phonological and semantic control deficits: A case-series comparison of Wernicke's aphasia, semantic dementia and semantic aphasia, Neuropsychologia, in press.

Rorden, C. (2005). MRIcro: http://www.sph.sc.edu/comd/rorden/mricro.html.

Saygin, A. P., Leech, R., \& Dick, F. (2010). Nonverbal auditory agnosia with lesion to Wernicke's area. Neuropsychologia, 48(1), 107-113.
Scott, S. K., Blank, C. C., Rosen, S., \& Wise, R. J. S. (2000). Identification of a pathway for intelligible speech in the left temporal lobe. Brain, 123, 2400-2406.

Seghier, M. L., Ramlackhansingh, A., Crinion, J., Leff, A. P., \& Price, C. J. (2008). Lesion identification using unified segmentation-normalisation models and fuzzy clustering. Neuroimage, 41(4), 1253-1266.

Shannon, R. V., Zeng, F. G., Kamath, V., Wygonski, J., \& Ekelid, M. (1995). Speech recognition with primarily temporal cues. Science, 270(5234), 303-304.

Tallal, P., \& Newcombe, F. (1978). Impairment of auditory-perception and language comprehension in dysphasia. Brain and Language, 5(1), 13-24.

Varney, N. R. (1984). Phonemic imperception in aphasia. Brain and Language, 21(1), 85-94.

Vigneau, M., Beaucousin, V., Herve, P. Y., Duffau, H., Crivello, F., Houde, O., et al. (2006). Meta-analyzing left hemisphere language areas: Phonology, semantics, and sentence processing. Neuroimage, 30, 1414-1432.

Vigneau, M., Beaucousin, V., Herve, P. Y., Jobard, G., Petit, L., Crivello, F., et al. (2011). What is right-hemisphere contribution to phonological, lexico-semantic and sentence processing? Insights from a meta-analysis. Neuroimage, 54(1), 577-593. 